\title{
REGENERAÇÃO DE UMA POPULAÇÃO NATURAL DE Araucaria angustifolia (ARAUCARIACEAE) ${ }^{1}$
}

\author{
Giovani Festa Paludo², Adelar Mantovani ${ }^{3}$ e Maurício Sedrez dos Reis ${ }^{4}$
}

\begin{abstract}
RESUMO - Estudos têm indicado baixa capacidade de regeneração em Araucaria angustifolia (Bertol.) Kuntze, baseados principalmente no baixo número de indivíduos regenerantes, não existindo estudos da dinâmica da regeneração natural. Dessa forma, o objetivo deste trabalho foi estudar a dinâmica da regeneração de plantas de uma população natural conservada de $A$. angustifolia sob uma floresta desenvolvida. Em uma parcela permanente de 5,1 ha, na Reserva Genética Florestal de Caçador (SC), foram avaliados, em três anos consecutivos, os indivíduos regenerantes ( $<5 \mathrm{~m}$ de altura) e discutidos os principais fatores responsáveis pela estrutura encontrada, sendo o padrão espacial analisado com a Função K, de Ripley. Foi encontrada variação no número de ingressantes entre os anos avaliados, bem como nos indivíduos mortos. O estágio crítico para a regeneração da espécie se mostrou logo nos primeiros anos de desenvolvimento até os 0,5 $\mathrm{m}$ de altura. A agregação predominou nos indivíduos ingressantes e na regeneração natural como um todo. Mas como a espécie é longeva, ela pode manter baixo número de indivíduos regenerantes na população e, mesmo assim, ter sucesso na regeneração.
\end{abstract}

Palavras-chave: Dinâmica florestal, Pinheiro-brasileiro e Mortalidade.

\section{REGENERATION IN A NATURAL POPULATION OF Araucaria angustifolia (ARAUCARIACEAE)}

\begin{abstract}
Some studies have been suggesting low regeneration capacity in Araucaria angustifolia (Bertol.) Kuntze, based mainly on the small number of regenerating individual. However, there are no works on natural regeneration dynamics. The objective of this work was to study regeneration dynmics of plants in a conservednatural population of A. angustifolia under a developed forest. In a permanent 5.1 ha-plot was installed in the Forest Genetic Reserve of Caçador, state of Santa Catarina. It was evaluated in three consecutive years the regenerating individuals ( $<5 \mathrm{~m}$ tall) and it was discussed the probable factors responsible for found structure. The spatial pattern was analyzed with the Ripley's K Function. It was found variation in the number of ingressing individuals among the evaluated years, as well as for the dead individuals. The critical stage of regeneration of this species appeared in the first years of development $0.5 \mathrm{~m}$ of height. Aggregation was predominant in the ingressing individuals and in natural regeneration as a whole. But, because A. angustifolia is a longlived species, it can keep a low number of regenerating individuals in the population and even though succeed in regeneration.
\end{abstract}

Keywords:Forest dynamic, Brazilian-pine and Mortality.

\section{INTRODUÇÃO}

A manutenção de populações naturais é dependente de sua regeneração natural, e conhecer essa característica torna-se fundamental para propor ações de recuperação das populações. Estudos da regeneração natural em Floresta Ombrófila Mista (FOM) tem encontrado baixos valores de densidade para Araucaria angustifolia (Bertol.) Kuntze quando comparada com as demais espécies (NARVAES et al., 2005) e casos em que não foram encontrados indivíduos na regeneração em ambiente com dominância da A. angustifolia no estrato superior (CALDATO et al., 1996). Na Floresta Nacional de São

\footnotetext{
${ }^{1}$ Recebido em 18.09.2009 e aceito para publicação em 02.05.2011.

${ }^{2}$ Graduação em Engenharia Florestal, Programa de Bolsas de Iniciação Científica da Universidade do Estado de Santa Catarina. E-mail: <giovanipaludo@yahoo.com.br>.

${ }^{3}$ Universidade do Estado de Santa Catarina, UDESC, Brasil. E-mail: <mantovani@cav.udesc.br>.

${ }^{4}$ Programa de Pós-Graduação em Ecologia da Universidade Federal de Santa Catarina.
} 
Francisco de Paula (FNSFP), em estudo de Duarte et al. (2002) foram raros os indivíduos da regeneração natural, bem como em estudo de Backes (1983) em Canela, RS. Souza et al. (2008), estudando a regeneração de A. angustifolia também na FNSFP, encontraram estrutura indicando deficiência na regeneração em floresta desenvolvida. Em contrapartida, ao estudar locais com histórico recente de corte, esses mesmos autores indicaram regeneração em pulsos de recrutamento. Também em florestas com longo histórico de perturbação, Sampaio e Guarino (2007) encontraram estrutura compatível com espécie que se regenera. Contudo, é interessante notar que, em estudo da dinâmica da FOM, Schaaf et al. (2006), utilizando indivíduos com diâmetro à altura do peito (DAP) $>20 \mathrm{~cm}$, verificaram que $A$. angustifolia aumentou sua participação na estrutura da floresta, mesmo sem apresentar estrutura de J-invertido. Por sua vez, Paludo et al. (2009), estudando uma população na Reserva Genética Florestal de Caçador (RGFC), encontraram baixa regeneração, mas compatível com uma espécie que se regenera sob floresta desenvolvida. Esses trabalhos em geral se basearam em levantamentos pontuais no tempo da população. Esse tipo de levantamento não traz informações seguras para inferir sobre a permanência da população no local. Para essa questão são necessários estudos de dinâmica, ou seja, avaliar a variação da população em um intervalo de tempo. Para toda a área de ocorrência não existem trabalhos publicados que tratam da dinâmica da regeneração natural de $A$. angustifolia .

Araucaria angustifolia é considerada como ameaçada, sendo a massiva coleta de sementes para consumo humano uma das causas (FARJON, 2006). Existe regulamentação para a coleta, mas sem embasamento ecológico necessário. Para estabelecer a quantidade de sementes que pode ser coletada sem prejudicar o processo de regeneração natural, primeiramente é necessário conhecer esse processo. Uma das formas de se fazer isso é através de estudos de dinâmica populacional, ou seja, estudando quantos indivíduos ingressam na população, quantos morrem, quanto é o crescimento e como estão distribuídos.

Questões que afetam a regeneração devem ser esclarecidas. Em trabalhos anteriores há indicações de que a fauna arranca indivíduos do estádio plântula (MELLO-FILHO et al., 1981; SANQUETTA et al., 2005) na busca pelas sementes que permanecem conectadas por certo período após a emergência. Outra questão é a queda de pinhas antes da época de maturação. Em anos anteriores, por observações pessoais foi encontrado no chão grande número de pinhas imaturas antes da época de maturação. Espera-se que esses eventos ocorram em intensidade importante a serem considerados como fatores, limitando o processo de regeneração da população e merecem ser estudados.

Algumas adaptações morfológicas e fisiológicas encontradas na A. angustifolia por Franco e Dillenburg (2007) foram sugeridas como ajustes a uma absorção mais eficiente de luz e conservação de recursos sob a sombra. Acredita-se que sob floresta desenvolvida a conservação de recursos seja estratégia necessária para o desenvolvimento e sobrevivência da regeneração natural. Dessa forma, é esperado que o indivíduo da regeneração natural deva manter relação proporcional de crescimento em altura e diâmetro para sua sobrevivência.

O objetivo deste trabalho foi avaliar a distribuição espacial, o crescimento e a dinâmica da regeneração natural de Araucaria angustifolia sob uma floresta desenvolvida em local conservado. Como podem ser fatores limitantes na regeneração da espécie foi procurado quantificar o número de eventos da queda de pinhas antes da maturação e o arranquio de plântulas na população. Além disso, foi procurado verificar as seguintes questões: se a desproporcionalidade do crescimento condiciona a morte do indivíduo sugere a existência de relação entre a mortalidade e a alometria; se o número de ingressantes é constante ao longo do tempo; e como estão distribuídos espacialmente esses ingressantes.

\section{MATERIAL E MÉTODOS}

\subsection{Espécie de estudo}

Araucaria angustifolia (Bertol.) Kuntze é uma gimnosperma de ocorrência na América do Sul, com área predominante na Região Sul do Brasil: sua distribuição vai do Estado do Rio Grande do Sul até o Sul de Minas Gerais (REITZ; KLEIN, 1966; REITZ et al., 1978; KLEIN, 1984). Popularmente conhecida como pinheiro-brasileiro, é uma espécie dioica, raramente monoica, anemófila e característica da FOM. Essa tipologia florestal foi reduzida a $12,6 \%$ da área de ocorrência original (RIBEIRO et al., 2009), e A. angustifolia foi bastante explorada em razão da qualidade da madeira (REITZ et al., 1978; RIZZINI, 1978). Esse cenário fez

Revista Árvore, Viçosa-MG, v.35, n.5, p.1107-1119, 2011

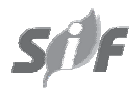


que a espécie fosse incluída como criticamente em perigo de extinção na lista da União Internacional para Conservação da Natureza (IUCN) (FARJON, 2006) e como vulnerável a extinção na Lista das Espécies da Flora Ameaçada de Extinção (BRASIL, 2008).

\section{1. Área de estudo}

O trabalho foi realizado em uma área pertencente à Reserva Genética Florestal de Caçador (RGFC), Estado de Santa Catarina. A reserva está localizada entre as latitudes $26^{\circ} 49^{\prime}$ e $26^{\circ} 53^{\prime}$ S e longitudes 5059' e 50 53' WG, compreendendo altitudes de 900 a 1.104 m e abrangendo uma área de 1.157,48 ha. O clima, segundo a classificação de Köppen, é do tipo Cfb, ou seja, mesotérmico subtropical úmido, sem estação seca e com verões frescos (KURASZ et al., 2008). Atualmente, a área representa um dos maiores remanescentes contínuos com vegetação característica da Floresta Ombrófila Mista no Estado. O histórico de exploração da área foi de corte seletivo há aproximadamente 80 anos, sendo considerada como mata secundária em avançado estado de sucessão/mata primária (PUCHALSKY et al., 2006).

\subsection{Obtenção e análise dos dados}

Demarcou-se uma parcela com dimensões de 170 x 300 m dentro da floresta em avançado estágio de sucessão, totalizando uma área de 5,1 ha. A área para instalação dessa parcela foi escolhida por se tratar de um local seguro para a manutenção da parcela permanente. A área da parcela foi dividida em 510 subparcelas de 10 x 10 m para facilitar as avaliações e mapeamento dos indivíduos de A. angustifolia. Todos os indivíduos de A. angustifolia dentro das 510 subparcelas foram mapeados e mensurados.

Os indivíduos com altura $\geq 5 \mathrm{~m}$ foram inventariados apenas para compor a tabela dinâmica e para comparação da densidade com a regeneração natural. No primeiro ano de estudo, as plantas com altura $\geq 5 \mathrm{~m}$ foram observadas quanto à existência de estruturas reprodutivas e classificadas como adultas quando presentes.

A regeneração natural, alvo deste trabalho, foi considerada como todos os indivíduos com altura $<5 \mathrm{~m}$ presentes nas 510 subparcelas de 10 x 10 m. A regeneração natural foi dividida em duas classes: regeneração $I$, indivíduos com altura $<1,5 \mathrm{~m}$; e regeneração II, indivíduos com altura $\geq 1,5 \mathrm{~m} \mathrm{e}<5 \mathrm{~m}$.
A regeneração I e a regeneração II foram inventariadas em três momentos: fevereiro de 2007, 2008 e 2009. Os novos indivíduos encontrados nos anos de 2008 e 2009 foram denominados ingressantes de 2008 e ingressantes de 2009, respectivamente. Em 2007, os indivíduos foram marcados e mensurados quanto à sua altura, bem como foram localizados dentro da parcela através de coordenadas $x$ e $y$. O DAP foi medido para a regeneração II. Em 2008, as plantas foram novamente inventariadas. As plantas mortas foram registradas, e as que continuavam vivas tiveram remedidos o DAP e a altura. Nesse ano foi medido o diâmetro do colo (DC) para a regeneração I, sendo em 2009 repetido o mesmo procedimento de 2008. Os ingressantes de 2008 e 2009 foram localizados na parcela, sendo mensurados o DC e a altura. As medições foram realizadas com o auxílio de suta, clinômetro, paquímetro e trena. Aqueles indivíduos que nos anos de 2008 e 2009 pertenciam à regeneração natural e cresceram a uma altura $\geq 5 \mathrm{~m}$ foram denominados recrutas.

Simultaneamente aos levantamentos demográficos de 2008 e 2009, foram contadas as pinhas encontradas no chão em todos os 5,1 ha. Os levantamentos ocorreram em fevereiro, período em que as pinhas em geral não estão maduras. Como o ciclo da produção de sementes é de aproximadamente 30 meses (MANTOVANI et al., 2004), eram encontradas pinhas cuja maturação se daria no mesmo ano ou no próximo ano do levantamento. Assim, as pinhas foram dividas em classes de acordo com a safra: 2008, 2009 ou 2010. O levantamento das plântulas arrancadas se baseou em uma avaliação, ocorrida em 2010 também em todos os 5,1 ha. Plântulas arrancadas foram consideradas como no primeiro estágio de vida após a germinação (para o conceito de plântula, ver SOUZA et al., 2008), que estavam no sentido horizontal sob a superfície do solo, não enraizadas no solo e ainda verdes.

Os cálculos de incremento de DC, altura e DAP foram realizados utilizando-se as medições das plantas que se mantiveram vivas entre os inventários de 2007 a 2009.

Para avaliar a conservação de recursos como estratégia de sobrevivência sob a floresta desenvolvida, foi investigado se a relação desproporcional de altura e diâmetro poderia ser uma das causas da morte de indivíduos. A relação alométrica foi, então, calculada entre os mortos e sobreviventes de 2009. Na impossibilidade de medição de indivíduos mortos, a 
alometria foi baseada na medição de altura e diâmetro (DC para regeneração I e DAP para regeneração II) dos indivíduos realizada no ano anterior (2008), tanto para os mortos quanto para os sobreviventes. Os dados foram transformados em logaritmo na base 10, sendo a regressão dos quadrados mínimos calculada em seguida. Relações alométricas foram, então, expressas pela equação $\mathrm{y}=\mathrm{a} \mathrm{x}^{\mathrm{b}}$, ou $\log \mathrm{y}=\log \mathrm{a}+\mathrm{b} \log \mathrm{x}$, em que y corresponde ao diâmetro (variável dependente) e x, à altura (variável independente). Diferenças entre as inclinações da regressão dos mortos e dos sobreviventes foram testadas pelo teste t (ZAR, 1999). Já o número de ingressantes dos anos de 2008 e 2009 foi comparado pelo teste do $\chi^{2}$ em nível de significância de $1 \%$.

Para a análise do padrão espacial foi utilizada a Função K-univariada de Ripley (RIPLEY, 1977). O raio (h) empregado para o cálculo do K(h) foi de $5 \mathrm{~m}$, sendo as análises feitas até a distância de 85 m, correspondente à metade do menor lado da parcela. Os valores de K(h) foram transformados através da função $\mathrm{L}(\mathrm{h})$ para melhor interpretação e visualização dos resultados. Os limites de confiança foram criados utilizando 499 simulações de eventos em completa aleatoriedade espacial. A Função $\mathrm{K}$ e os respectivos intervalos de confiança foram calculados utilizando-se algoritmos escritos no R ( $R$ DEVELOPMENT CORE TEAM, 2009) pacote splancs (ROWLINGSON; DIGGLE, 1993).

\section{RESULTADOS}

\subsection{Dinâmica da regeneração}

Em 2007, a população apresentou densidade de 104 ind.ha- ${ }^{-1}(n=531)$. Dos indivíduos que foram avaliados apenas em 2007, ou seja, aqueles com a altura $\geq 5 \mathrm{~m}$, representaram 63 ind.ha- ${ }^{-1}(n=322)$. Dos quais, 28 ind.ha-1 ( $n=145$ ) eram indivíduos reprodutivos e 35 ind.ha ${ }^{-1}$ (n = 177), indivíduos não reprodutivos. Já a regeneração natural, avaliada por três anos, apresentou número médio de indivíduos de 48 ind.ha ${ }^{-1}(\mathrm{n}=245)$. O número de ingressantes não foi constante nos dois anos analisados $\left(\chi^{2}=26,3 ; \mathrm{P}<0.001 ; \mathrm{GL}=1\right)$, otendo-se 25 ind.ha ${ }^{-1}(\mathrm{n}=125)$ e 11 ind.ha ${ }^{-1}(\mathrm{n}=56)$ nos anos de 2008 e 2009, respectivamente (Tabela 1).

A distribuição de alturas para todos os indivíduos da regeneração natural, quando comparada nos três anos, apresentou maiores variações apenas na primeira classe de altura (Figura 1).

Ingressantes em 2008 apresentaram DC médio de $0,28 \mathrm{~cm}(\mathrm{~s}=0,073 \mathrm{~cm})$ e altura média de 23,0 cm (s = 9,30 cm) e em 2009, DC médio de 0,26 cm $(\mathrm{s}=0,11 \mathrm{~cm})$ e altura média de $24,0 \mathrm{~cm}(\mathrm{~s}=8,4 \mathrm{~cm})$, não diferentes entre os dois anos avaliados em DC $(t=1,34 ; p=0,18)$ e em altura $(t=-0,49 ; p=0,63)$.

A mortalidade predominou na primeira classe de altura (até $50 \mathrm{~cm}$ ) nos dois anos avaliados, conforme a Figura 2A. As demais classes apresentaram baixo número de indivíduos mortos. Dos 124 indivíduos mortos no levantamento de 2009, 99 foram ingressantes de 2008.

Durante o levantamento de 2009 foram encontradas 15 plantas arrancadas ainda verdes em toda a parcela, perfazendo aproximadamente 3 ind.ha ${ }^{-1}$, que ingressariam na população em 2009. Em 2008 foram encontradas 96 pinhas, ou seja, 19 pinhas.ha ${ }^{-1}$, imaturas (88 da safra de 2008 e 8 da safra de 2009) e em 2009, 168, perfazendo 33 pinhas.ha-1 (165 da safra de 2009 e 3 da safra de 2010).

A regressão foi feita apenas para a regeneração I, já que da regeneração II apenas cinco indivíduos morreram em 2009. Tanto a equação da regressão dos sobreviventes $\left(\mathrm{y}=0,02910 *(\text { altura })^{0,7273} ; \mathrm{r}^{2}=0,839\right)$ quanto a dos mortos $\left(\mathrm{y}=0,07760 *\right.$ (altura $^{0,4198}$;

Tabela 1 - Dinâmica da regeneração natural de Araucaria angustifolia em uma parcela de 5,1 ha na Reserva Genética Florestal de Caçador, SC.

Table 1 - Natural regeneration dynamics of Araucaria angustifolia in a 5.1 ha-plot in the Forest Genetic Reserve of Caçador, SC.

\begin{tabular}{|c|c|c|c|c|c|c|c|c|c|}
\hline & & \multicolumn{5}{|c|}{ Regeneração natural (< 5 m de altura) } & \multicolumn{3}{|c|}{$\geq 5 \mathrm{~m}$ de altura } \\
\hline & & & Altura & Altura & & & & & \\
\hline Ano & População & Ingressantes & $<1,5 \mathrm{~m}$ & $\geq 1.5 \mathrm{e}<5 \mathrm{~m}$ & Mortos & Total & Recrutas & Mortos & Total \\
\hline 2007 & 531 & - & 161 & 48 & - & 209 & - & - & 322 \\
\hline 2008 & 615 & 125 & 128 & 45 & 35 & 298 & 1 & 6 & 317 \\
\hline 2009 & 543 & 56 & 126 & 46 & 124 & 228 & 2 & 4 & 315 \\
\hline média & 563 & & 138 & 46 & & 245 & & & 318 \\
\hline
\end{tabular}

Revista Árvore, Viçosa-MG, v.35, n.5, p.1107-1119, 2011 
$\left.\mathrm{r}^{2}=0,320\right)$ mostraram relações alométricas significativas entre a altura $(\mathrm{cm})$ e o DC $(\mathrm{cm})(\mathrm{P}<0,001)$. O coeficiente angular dos sobreviventes diferiu significativamente do coeficiente angular dos mortos (teste $\mathrm{t}=4,89$; $\mathrm{P}$ $<0,001)$.

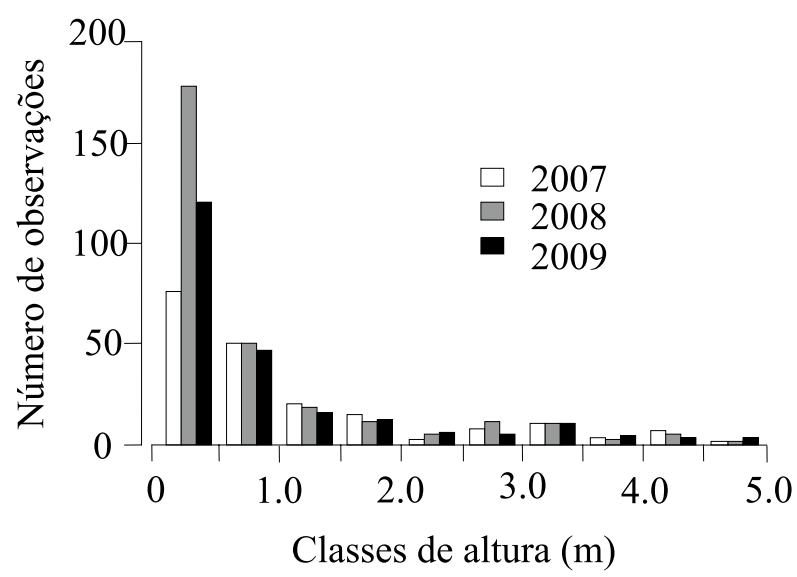

Figura 1 - Distribuição de alturas dos indivíduos da regeneração natural (altura $<5 \mathrm{~m}$ ) de Araucaria angustifolia, nos três anos avaliados na Reserva Genética Florestal de Caçador, SC.

Figure 1 -Distribution of individual heights in natural regeneration (height $<5 \mathrm{~m}$ ) of Araucaria angustifolia, in the Forest Genetic Reserve of Caçador, SC.

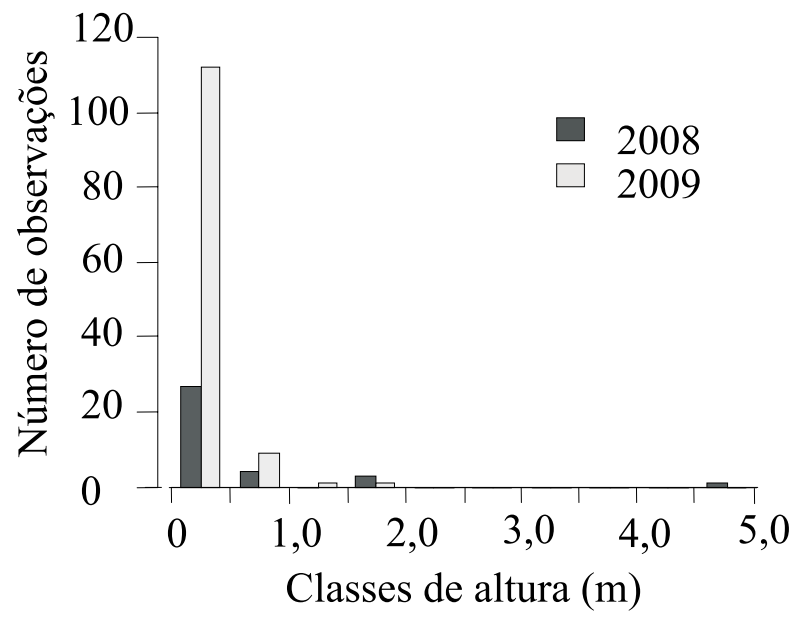

Figura 2-Distribuição da mortalidade de Araucaria angustifolia em 2008 e 2009 na Reserva Genética Florestal de Caçador, SC.

Figure 2 - Araucaria angustifolia mortality distribution in 2008 and 2009 in the Forest Genetic Reserve of Caçador, SC.

\subsection{Crescimento}

A duas classes da regeneração juntas apresentaram crescimento médio anual, entre 2007 e 2009, em altura de $6,90 \mathrm{~cm}(\mathrm{~s} \pm 12,0 \mathrm{~cm})$. A regeneração I teve crescimento médio anual de altura entre 2007 e 2009 de 4,30 cm $(\mathrm{s} \pm 6,60 \mathrm{~cm})$ e DC de $0,0653 \mathrm{~cm}(\mathrm{~s} \pm 0,181 \mathrm{~cm})$. A regeneração 2 teve incremento médio anual em DAP de $0,040 \mathrm{~cm}(\mathrm{~s} \pm 0,25 \mathrm{~cm})$ e altura de $13,4 \mathrm{~cm}(\mathrm{~s} \pm 18,6 \mathrm{~cm})$.

\subsection{Padrão espacial}

Os indivíduos que ingressaram em 2008 apresentaram agregação até a escala de aproximadamente 55 m (Figura 3A). Já naqueles ingressantes em 2009 a agregação apareceu em toda a escala analisada (Figura 3B).

Os padrões espaciais da mortalidade diferiram nos dois anos avaliados. Enquanto em 2008 os indivíduos mortos apresentaram predominantemente aleatoriedade no arranjo espacial (Figura 3C), os indivíduos mortos de 2009 amostraram predominância do padrão agregado (Figura 3D).

O padrão espacial da regeneração natural apresentou pouca variação entre os anos (Figura 4). Um pico na intensidade da função apareceu nos três anos avaliados nas escalas entre 20 e 25 m, e a agregação manteve-se na escala entre 50 e $60 \mathrm{~m}$.

\section{DISCUSSÃO}

\subsection{Dinâmica da regeneração}

Encontrou-se variação no número de indivíduos que ingressaram na população nos dois anos avaliados. Essa variação pode ser o resultado da alternância de produção de sementes conhecida em A. angustifolia, como mencionado por Mantovani et al. (2004), e semelhante ao observado em Araucaria araucana (MONTALDO, 1974). A variação anual na remoção de sementes pela fauna (IOB; VIEIRA, 2008) também pode ter influências no número de indivíduos ingressantes. $\mathrm{E}$, ainda, o fato de que as sementes de A. angustifolia constituem alimento para várias espécies da fauna silvestre (MELLO-FILHO et al., 1981; IOB; VIEIRA, 2008) torna mais difícil encontrar resposta direta à variação no número de ingressantes.

Neste trabalho, encontrou-se grande parte da mortalidade concentrada na classe até $50 \mathrm{~cm}$ de altura (Figura 2A), semelhante ao observado por Backes (2001), através de avaliação pontual no Rio Grande do Sul,

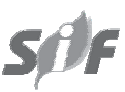

Revista Árvore, Viçosa-MG, v.35, n.5, p.1107-1119, 2011 
corroborando que esse seja um estágio crítico para a regeneração natural da espécie.

Entre os possíveis fatores críticos para a regeneração de uma espécie estão a intensidade de luz, a qualidade de luz, o nível de competição das raízes, pulsos de nutrientes, textura do solo e efeito de predadores e patógenos (CLARK; CLARK, 1987). É possível levantar alguns fatores mais evidentes em $A$. angustifolia que podem provocar a mortalidade das plantas ingressantes na área de estudo: (1) alta procura por sementes de
A

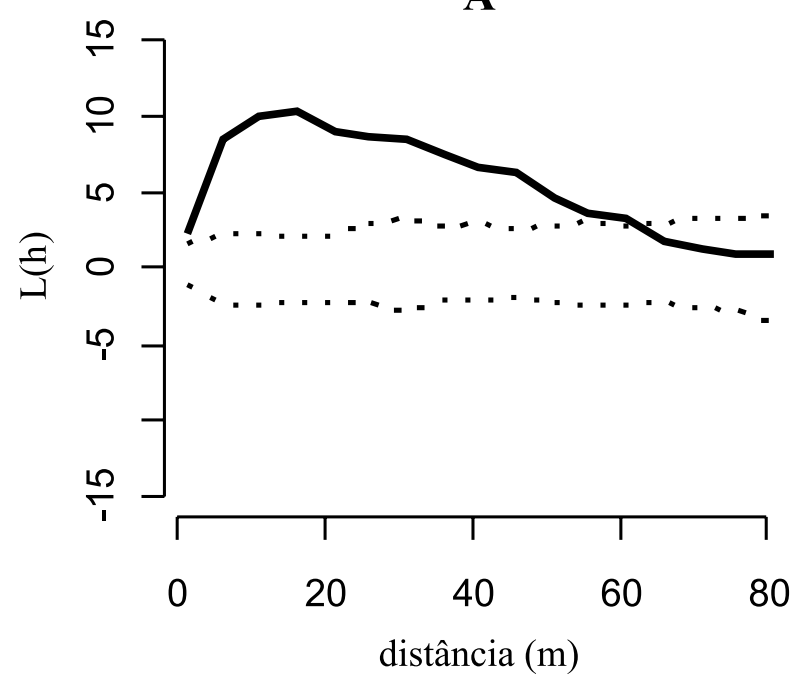

C

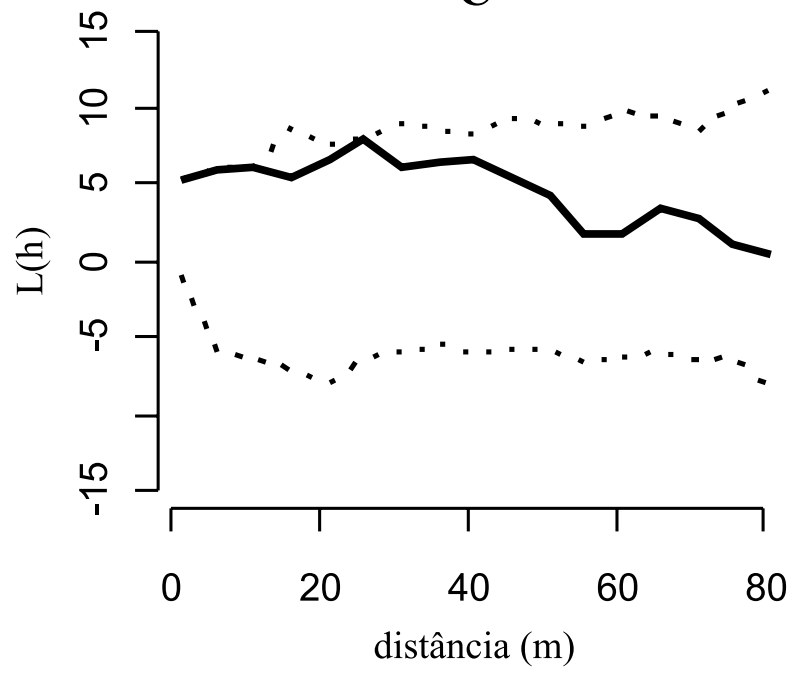

B

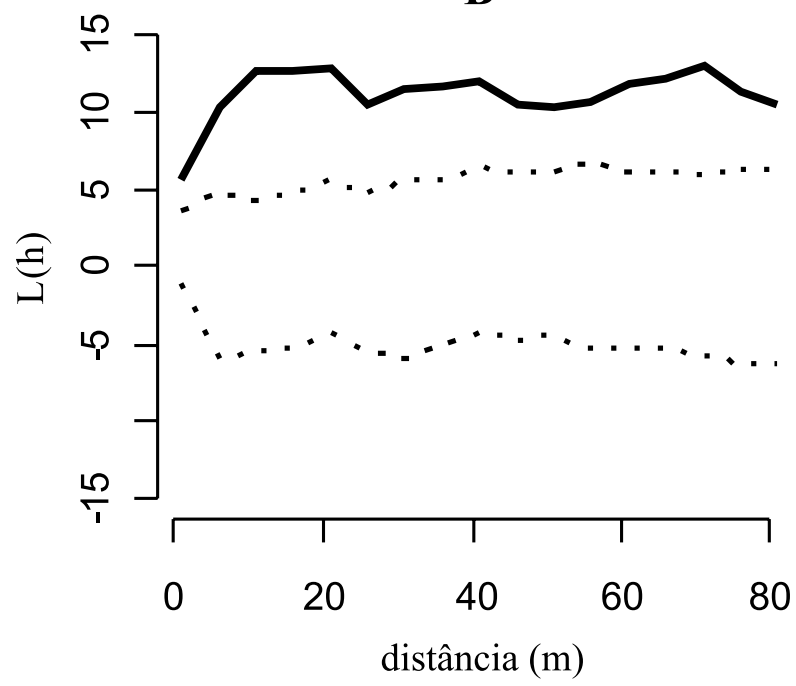

D

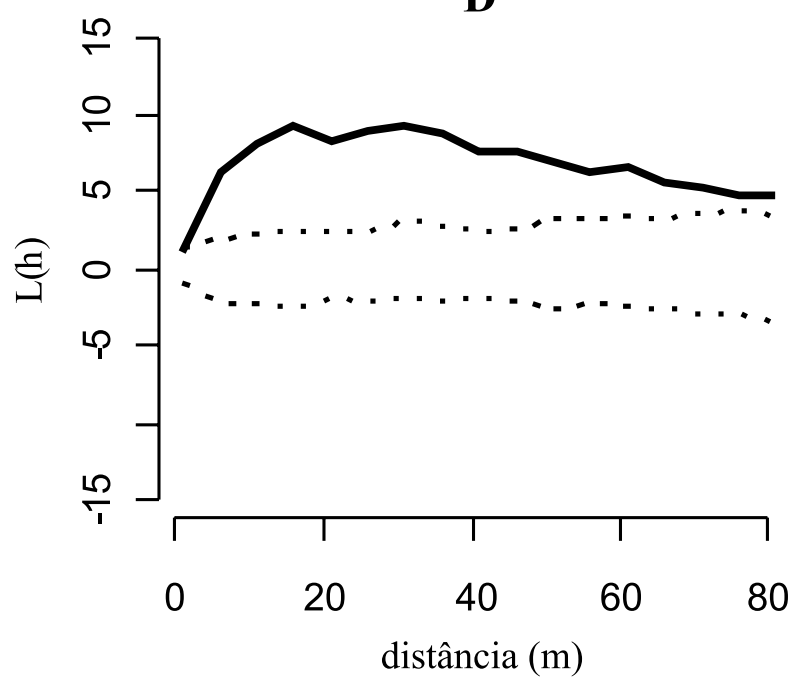

Figura 3 - Análise do padrão espacial para ingressantes de 2008 (A) e 2009 (B) e mortos de 2008 (C) e 2009 (D), em uma população natural de Araucaria angustifolia na Reserva Genética Florestal de Caçador, SC, através da função K de Ripley. Linhas contínuas são os resultados da função K transformada em L(h). Linhas pontilhadas representam os intervalos de confiança (envelopes) construídos com 499 simulações de completa aleatoriedade espacial.

Figure 3 - Spatial pattern analysis through Ripley's K Function for new individuals from 2008 (A) and 2009 (B) and dead individuals from 2008 (C) and 2009 (D) in a natural population of Araucaria angustifolia in the Forest Genetic Reserve of Caçador, SC. The continuous lines are the results of $K$ function transformed into $L(h)$. The spotted lines are de confidence limits (envelopes) computed with 499 simulations of complete spatial randomness.

Revista Árvore, Viçosa-MG, v.35, n.5, p.1107-1119, 2011 


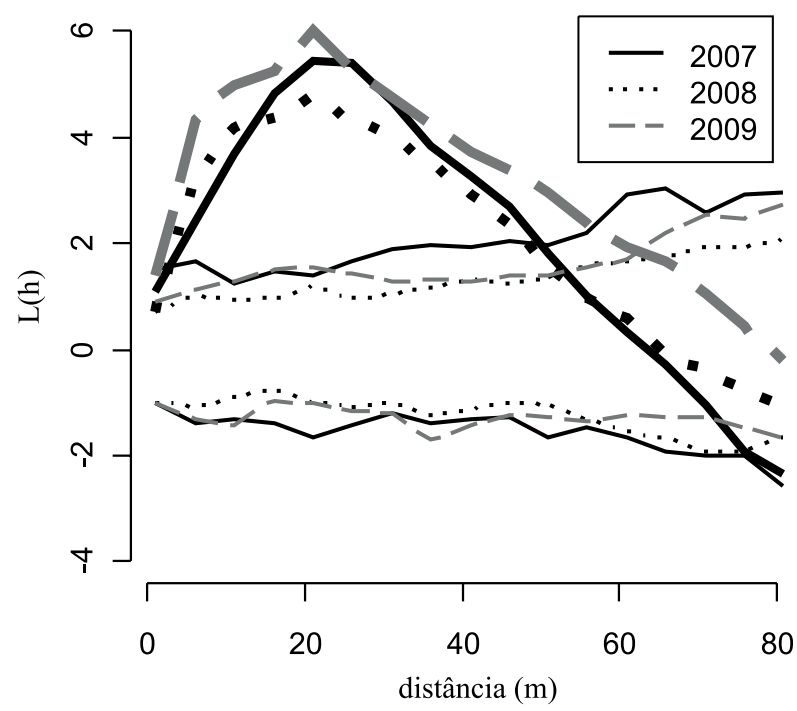

Figura 4 - Variações na análise do padrão espacial da regeneração natural em três diferentes anos, em uma população natural de Araucaria angustifolia na Reserva Genética Florestal de Caçador, SC. Linhas largas representam a função transformada do K de Ripley. Linhas finas representam os intervalos de confiança (envelopes) construídos com 499 simulações de completa aleatoriedade espacial.

Figure 4 - Variations in the spatial pattern analysis in three different years in a natural population of Araucaria angustifolia in the Forest Genetic Reserve of Caçador, $S C$. Broad lines are transformed Ripley's K function. Thin lines are the confidence limits (envelopes) computed with 499 simulations of complete spatial randomness.

A. angustifolia pela fauna (MULLER; MACEDO, 1980; IOB; VIEIRA, 2008) faz que plântulas sejam também arrancadas (MELLO-FILHO et al., 1981; SANQUETTA et al., 2005); durante o levantamento de 2009, foram encontradas 15 plântulas não enraizadas, com características de terem sido recentemente arrancadas - essas plântulas em geral não possuíam os cotilédones e a gema apical, sugerindo a herbivoria; (2) o folhedo de $A$. angustifolia pode representar aproximadamente $60 \%$ do folhedo total produzido pelas espécies da FOM, conforme estudo de Backes et al. (2005) em outra área, e seu acúmulo dá-se principalmente sob as copas de A. angustifolia, podendo cair sobre as plântulas e matando-as; e (3) o efeito da presença de outras espécies, como gramíneas com densa cobertura na superfície do solo e que podem interferir no sucesso do estabelecimento.
Em contrapartida, outros fatores parecem ter pouca ou nenhuma influência da floresta estudada na mortalidade das plantas ingressantes. O pastoreio não existia na área do estudo, embora também não tenha sido encontrado padrão da resposta de populações de A. angustifolia ao pastoreio (SAMPAIO; GUARINO, 2007).

Duarte et al. (2002) verificaram que a disponibilidade de luz não limitava a regeneração de $A$. angustifolia, assim como indicado por Inoue et al. (1978) e Duarte e Dillenburg (2000) para plântulas em estudos realizados em casa de vegetação. Estudos sobre o crescimento e, ou, mortalidade e sua relação com a luz, no ambiente natural, poderiam esclarecer melhor o papel da luz na dinâmica de A. angustifolia .

Nos levantamentos de 2008 e 2009 foram encontradas 96 e 168 pinhas imaturas no chão, respectivamente. Estudos indicam que o pico de maturação e queda de sementes ocorrem nos meses de abril e maio (REITZ et al., 1978; FASSOLA et al., 1999; MANTOVANI et al., 2004), mas, conforme as variedades descritas por Reitz e Klein (1966), iniciam-se em fevereiro, chegando até dezembro. Os levantamentos foram realizados no mês de fevereiro, e assim as sementes nas pinhas derrubadas ainda estavam imaturas. Avaliando a produção de pinhão na RGFC, Silva e Reis (2009) encontraram a produção de 5,6 pinhas por indivíduo feminino (em 2004 e 2005). Na RGFC, indivíduos femininos ocorrem em densidade de 13,9 ind.ha ${ }^{-1}$ (PALUDO et al., 2009). Pelo cálculo, isso resultaria numa produção de 78 pinhas.ha-1 ${ }^{-1}$,6 pinhas.fêmea ${ }^{-1}$ multiplicadas por 13,9 fêmea.ha-1). Como em 2009 foram encontradas 33 pinhas.ha ${ }^{-1}$ derrubadas antes da safra, essa é uma questão importante a ser considerada no processo de regeneração da espécie. Macacos como o bugio (Allouata sp.) e o macaco-prego (Cebus sp.) podem ser apontados como um dos prováveis fatores da derrubada prematura de pinhas, como foi visto durante um dos levantamentos realizados, embora outros fatores, como o vento, podem estar envolvidos. Além disso, uma das causas apontadas para falta ou reduzida quantidade de regenerantes é a massiva coleta de sementes por humanos (SOUZA, 2007), o que ocorre também em unidades de conservação (NEGRELLE; LEUCHTENBERGER, 2001; PALUDO et al., 2009). Na área de estudo também há coleta, mas aparenta ser de baixa intensidade, o que provavelmente afeta pouco a quantidade de regenerantes, embora essa questão não tenha sido testada e quantificada.

Revista Árvore, Viçosa-MG, v.35, n.5, p.1107-1119, 2011 
Quando adulta, A. angustifolia ocupa o estrato superior da floresta (KLEIN, 1980; KLEIN, 1984), caracterizando a necessidade de luz solar plena. Para alcançar esse estrato, a espécie pode seguir um dos modelos de regeneração proposto por Clark e Clark (1987) no qual a espécie pode necessitar de uma clareira no estado juvenil para alcançar o dossel rapidamente em pulsos de crescimento antes que a clareira seja ocupada por outras espécies, ou seja fechada pelas copas. Algumas adaptações morfológicas e fisiológicas encontradas na $A$. angustifolia (entre elas o crescimento inicial maior em altura) por Franco e Dillenburg (2007) foram sugeridas como ajustes a uma absorção mais eficiente de luz e conservação de recursos sob a sombra, suportando a ideia da tolerância ao sombreamento em parte do processo de regeneração. Neste trabalho, as relações alométricas entre as medições de 2008 calculadas com os mortos e sobreviventes de 2009 apoiaram essa ideia. Os sobreviventes apresentaram coeficiente alométrico significativamente maior do que os mortos, ou seja, existe maior proporcionalidade nas variações de diâmetro em relação às variações em altura nos indivíduos sobreviventes. Como nesse estágio o indivíduo precisa crescer em altura, então o resultado sustenta a ideia de que o crescimento proporcional de DC e a altura são uma forma de conservação de recursos. Já o crescimento desproporcional aumenta as chances de o indivíduo morrer. Contudo, é bastante provável que vários outros fatores estejam envolvidos na mortalidade da espécie, e a análise é temporalmente restrita. Esse padrão deveria ser testado em plantas de classes maiores e durante vários anos para confirmar que a relação alométrica possa ser indicativo no processo de regeneração.

Em diferentes regiões da FOM, A. Angustifolia ocorre em densidades que vão de 10 a até 138 ind.ha $^{-1}$ (NASCIMENTO et al., 2001; NEGRELLE; LEUCHTENBERGER, 2001; GERALDI etal., 2005; SEGER et al., 2005; CORDEIRO; RODRIGUES, 2007). Na RGFC, A. angustifolia mostrou-se como a espécie mais abundante e dominante nos estratos superiores (NEGRELLE; SILVA, 1992) e em levantamento fitossociológico também na RGFC. Silva et al. (1997), considerando DAP $>20 \mathrm{~cm}$ e utilizando como critério de seleção de unidades amostrais a presença de $A$. angustifolia, encontraram 53,8 ind.ha ${ }^{-1}$. Neste trabalho, a parcela de 5,1 ha indivíduos com altura $\geq 5 \mathrm{~m}$ representa 63 ind.ha ${ }^{-1}$ (Tabela 1). Mas na regeneração natural da
FOM várias espécies arbóreas aparecem em densidade superior a 200 ind.ha ${ }^{-1}$, chegando algumas plantas a 1.000 ind.ha ${ }^{-1}$, dependendo do critério de exclusão utilizado no levantamento (CALDATO et al., 1996; NARVAES et al., 2005).

Considerando que a regeneração natural da $A$. angustifolia ocorre através da formação de banco de plântulas, como é indicado por Duarte et al. (2002), seria esperada proporção maior de regenerantes do que adultos. Em A. angustifolia, Sampaio e Guarino (2007) encontraram maior número de plantas com DAP $<5 \mathrm{~cm}$ do que adultos em cinco populações estudadas. Neste trabalho, foi constatado 48 ind.ha ${ }^{-1}$ com altura $<5$ m, semelhante ao encontrado por Souza et al. (2008) na FNSFP, onde indivíduos com altura $<8$ m apresentaram densidade média de 51,4 ind.ha ${ }^{-1}$, variando de 9 a 132 ind.ha ${ }^{-1}$, variação essa não relacionada ao histórico de manejo. Puchalsky et al. (2006), comparando nove populações no Estado de Santa Catarina, encontraram menor proporção de regenerantes na RGFC. Esses autores evidenciaram a proporção de regenerantes como indicador do grau de perturbação, embora as amostras tenham sido feitas com parcelas de 40 x $40 \mathrm{~m}$, o que pode comprometer a amostragem de regenerantes na RGFC, conforme o padrão espacial agregado encontrado por Paludo et al. (2009). O mesmo aconteceu com Caldato et al. (1996), que utilizaram parcelas de 10 x $25 \mathrm{~m}$, em estudo também na RGFC. Nesta pesquisa, encontrou-se aumento no número de regenerantes, o que suporta a hipótese diferente do que tem sido sugerido por alguns autores. Acredita-se que em áreas com maior grau de perturbação A. angustifolia tenha regeneração mais expressiva, promovendo a colonização da espécie, e que em florestas desenvolvidas sua regeneração seja baixa, mas o suficiente para manter sua estrutura demográfica. Ainda, A. angustifolia possui distribuição disjunta em menores latitudes no Estado de São Paulo até Minas Gerais e em menores altitudes nas zonas de transição entre a FOM e demais tipologias florestais no Estado de Santa Catarina, assim como a distribuição disjunta dos campos dentro dos limites da FOM é argumento que sustenta o avanço da espécie sobre campos e o seu recuo nas áreas de transição dessa tipologia florestal com as demais (REITZ; KLEIN, 1966; KLEIN, 1978). E isso pode fazer que o comportamento da regeneração ainda sofra variações de acordo com a situação e posição geográfica da população. Em adição, a predação de sementes é menor em áreas de campo 
aberto, quando comparada com o interior e a borda da floresta (IOB; VIEIRA, 2008), o que constitui outro fator, facilitando seu processo de avanço em áreas abertas de campo e a maior dificuldade de regeneração em floresta desenvolvida.

Considerando ainda que, por se tratar de espécie dioica com a razão sexual em geral 1:1 (BANDEL; GURGEL, 1967; PINTO, 1990; PALUDO et al., 2009), apenas metade dos indivíduos produz sementes, o que pode colaborar com menor número de regenerantes em relação ao número de indivíduos adultos, em comparação com o número de regenerantes de espécies monoicas.

Em suma, A. angustifolia é uma espécie longeva e mantém indivíduos em classes de diâmetros relativamente altas quando comparadas com angiospermas (SOUZA, 2007), e o seu estoque de regeneração pode ser representado por pequeno número na estrutura pontual (estática), mas ao longo dos anos o estoque pode ser suficiente para manter a estrutura demográfica da espécie.

Indivíduos de A. angustifolia com DAP de 70 a $90 \mathrm{~cm}$ ou de 90 a $110 \mathrm{~cm}$ chegam a mais de 100 anos (MATTOS et al., 2007). Talvez essa espécie, por ser longeva, possa se regenerar em episódios em ciclos de vários anos, como sugerido por Souza et al. (2008) e como pode ocorrer em outras espécies longevas, a exemplo de Nothofagus pumilio, no Chile (CUEVAS, 2002). A partir da premissa de episódios massivos para a regeneração, o fogo teria papel importante como sugerido por Soares (1979) em A. angustifolia. No entanto, a fragilidade das plantas regenerantes que crescem sob floresta desenvolvida (REITZ; KLEIN, 1966) e seu crescimento relativamente lento nas fases iniciais parecem não sustentar essa hipótese. Já o evento da seca dos taquarais (do gênero Merostachis) pode sustentar a ideia de regeneração em episódios, o que poderia ser testado, sobretudo, pelo fato de que os grupos de regeneração apresentam diâmetros semelhantes aos grupos formados pelas taquaras.

\subsection{Crescimento}

Os indivíduos regenerantes apresentaram baixo crescimento médio anual em altura: 6,9 cm. Essas medições apresentaram grande variação, evidenciada pelo desviopadrão das medidas. Em alguns casos, as plantas, ou os ramos, encurvavam-se, indicando senescência e perda de vigor e causando redução na altura total da planta, ou seja, decrescimento. O que foi encontrado neste trabalho corrobora os achados de Reitz e Klein (1966), que citaram que o desenvolvimento da $A$. angustifolia se processa de modo relativamente lento, sobretudo na fase inicial, em comparação com outras coníferas exóticas. É interessante ressaltar que o crescimento lento ou muito lento e a tolerância à sombra em estágios juvenis caracterizam uma espécie de bosque clímax (BUDOWSKI, 1965 apud CLARK; CLARK, 1987). Outras medições como a de DC apresentaram grande desvio-padrão, comprometendo inferências confiáveis.

\subsection{Padrão espacial}

Nos indivíduos que ingressaram em 2008, a agregação manteve-se até as escalas de 55 m, enquanto os que ingressaram em 2009 apresentaram agregação em todas as escalas. Esses resultados diferem daqueles de Souza et al. (2008), segundo os quais, de 10 parcelas analisadas, sete apresentaram arranjo aleatório, e o agregado apareceu em três parcelas, uma com a escala de agregação próxima a $50 \mathrm{~m}$, e as outras duas, nas quais a agregação apareceu em menores escalas.

O padrão espacial dos indivíduos que morreram em 2008 foi predominantemente aleatório. Se a mortalidade não é agregada, então os fatores causadores da mortalidade não agem em grupos, o que seria esperado se ela fosse devida a um patógeno. Em 2009, a mortalidade ocorreu intensamente agregada, o que pode ser explicado pelo próprio padrão espacial dos ingressantes de 2008, pois morreram 124 indivíduos, dos quais 99 eram ingressantes de 2008.

O padrão espacial da regeneração natural mantém-se ao longo dos anos, já que não apresentou grandes variações nos três anos avaliados (Figura 4). Um pico na intensidade da função apareceu nos três anos avaliados nas escalas entre 20 e 25 m, e a agregação manteve-se entre a escala de 50 e $60 \mathrm{~m}$. O conhecimento desse padrão é importante para a tomada de decisão na escolha do processo de amostragem em trabalhos com sua regeneração natural e também para entender os possíveis processos ecológicos responsáveis pelo padrão. Um padrão agregado exige um processo sistemático de amostragem. Ou, ainda, é possível a identificação dos agregados da regeneração natural na floresta, e a amostragem por esses grupos de regeneração também poderá ser feita. $\mathrm{O}$ padrão agregado da regeneração já foi encontrado em A. angustifolia por Souza et al. (2008), estudando plantas de até $6 \mathrm{~cm}$ de DAP e $8 \mathrm{~m}$ de altura, e em duas unidades amostrais

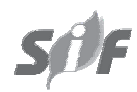

Revista Árvore, Viçosa-MG, v.35, n.5, p.1107-1119, 2011 
esses autores encontraram agregação até a escala de 30 e $50 \mathrm{~m}$ e em quatro unidades amostrais, arranjo aleatório. Veblen (1982) obteve padrão agregado em A. araucana nas classes de 9, 18 e 27 m de distância em diferentes sítios, classes de distâncias essas menores do que as encontradas neste trabalho. Esse mesmo autor considerou a regeneração natural como indivíduos com altura $>2$ m e $<10$ cm de DAP. Já Rigg (2005) encontrou predominantemente o padrão agregado em plantas de Araucaria laubenfelsii com altura $>1 \mathrm{~m}$. A análise de Rigg (2005) se deu até 25, 40 e 50 m. Conforme Paludo et al. (2009) e com as informações deste trabalho, a regeneração de A. angustifolia aparece agregada em escalas superiores a $50 \mathrm{~m}$, chegando até os aproximados $60 \mathrm{~m}$ (Figura 4), mas essa escala pode ser diferente da verdadeira, em razão da natureza acumulativa da função K d Ripley (PERRY et al., 2006). Rigg (2005) sugeriu que a agregação da regeneração foi resultado da limitada dispersão das sementes e de outros fatores como fogo e ciclones. Em A. angustifólia, a limitada dispersão parece não ser problema para a regeneração da espécie (PALUDO et al., 2009).

É interessante notar que outros fatores não estudados neste trabalho podem estar causando ou influenciando a agregação da população, como fatores morfológicos, ambientais e fitossociológicos (DALE, 1999).

\section{CONCLUSÕES}

A fase crítica de desenvolvimento da regeneração de Araucaria angustifolia é até $50 \mathrm{~cm}$ de altura, e a mortalidade estende-se até a classe de $2 \mathrm{~m}$ de altura. A quantificação das pinhas caídas antes da época de maturação se mostrou como fator que deve ser considerado no processo de regeneração da espécie. Os resultados atestam a viabilidade de regeneração da espécie mesmo sob floresta desenvolvida, já que foi encontrado um número crescente de regenerantes. No entanto, o acompanhamento da dinâmica da regeneração natural deve ser feito durante vários anos, para a obtenção de resultados mais consistentes, especialmente devido à grande longevidade da espécie.

\section{AGRADECIMENTOS}

Ao PROBIC e ao CNPq, pela concessão de bolsa aos autores; à FAPESC, pelo financiamento do Projeto; à EPAGRI/Caçador-SC, pela autorização do uso da área do experimento e alojamentos; a Alison Paulo Bernardi,
Camila Lucas Chaves e Cristiano Teixeira, pelas contribuições no desenvolvimento do trabalho; e ao Núcleo de Pesquisa em Florestas Tropicais - NPFT/ UFSC, pela participação e auxílio no Projeto.

\section{REFERÊNCIAS}

BACKES, A. Dinâmica do pinheiro brasileiro. Iheringia Serie Botanica, v.30, n.1, p.49-84, 1983.

BACKES, A. Determinação da idade e regeneracao natural de uma populacao de Araucaria angustifolia (Bertol) Kuntze em um povoamento ûorestal localizado no município de Caxias do Sul, RS, Brasil. Iheringia Serie Botanica, v.56, p.115-130, 2001.

BACKES, A.; PRATES, F. L.; VIOLA, M. G. Produção de serapilheira em Floresta Ombrófila Mista, em São Francisco de Paula, Rio Grande do Sul, Brasil. Acta Botanica Brasileira, v.19, n.1, p.155-160, 2005.

BANDEL, G.; GURGEL, J. T. A. Proporção do sexo em pinheiro brasileiro Araucaria angustifolia (Bert.) O. Kuntze. Silvicultura em São Paulo, v.6, p.209-220, 1967.

BRASIL. Ministério do Meio Ambiente. Instrução Normativa 06 de setembro de 2008. Brasília: ICMBIO. 2008

CALDATO, S. L. et al. Estudo da regeneração natural, banco de sementes e chuva de sementes na Reserva Genética de Caçador, SC. Ciência Florestal, v.6, n.1, p.27-38, 1996.

CLARK, D. A.; CLARK, D. B. Analisis de la regeneracion de arboles del dosel en bosque muy húmedo tropical aspectos teoricos y praticos. Revista Biologia Tropical, v.35, n.1, p.41-54, 1987.

CORDEIRO, J.; RODRIGUES, W. A. Caracterização ûtossociológica de um remanscente de ûoresta ombróûla mista em Guarapuava, PR. Revista Árvore, v.31, n.3, p.545-554, 2007.

CUEVAS, J. G. Episodic regeneration at the Nothofagus pumilio alpine timberline in Tierra del Fuego, Chile. Journal of Ecology, v.90, n.1, p.52-60, 2002. 
DALE, M. R. T. Spatial pattern analysis in plant ecology. Cambridge: Cambridge University Press, 1999.

DUARTE, L. S.; DILLENBURG, L. R. Ecophysiological responses of Araucaria angustifolia (Araucariaceae) seedlings to different irradiance levels. Australian Journal of Botany, v.48, p.531-537, 2000.

DUARTE, L. S.; DILLENBURG, L. R.; ROSA, L. M. G. Assessing the role of light availability in the regeneration of Araucaria angustifolia.

Australian Journal of Botany, v.50, n.6, p.741-751, 2002.

FARJON, A. 2006. Araucaria angustifolia. In: IUCN 2008. 2008 IUCN Red List of Threatened Species. Disponível em: <www.iucnredlist.org> Acesso em: 08 May 2009.

FASSOLA, H. E. et al. Observaciones sobre la producción de frutos e semillas en plantaciones de Araucaria angustifolia (Bert) O. K. (período 1993-1998). INTA EEA Informe Técnico, v.24, p.1-12, 1999.

FRANCO, A. M. S.; DILLENBURG, L. R. Ajustes morfologicos e ûsiologicos em plantas jovens de Araucaria angustifolia (Bertol) Kuntze em resposta ao sombreamento. Hoehnea, v.34, n.2, p.135-144, 2007.

GERALDI, S. E.; KOEHLER, A. B.; KAUANO, E. E. Levantamento ûtossociológico de dois fragmento da Foresta Ombróûla Mista, em Tijucas do Sul, PR. Revista Acadêmica de Curitiba, v.3, n.2, p.27-36, 2005.

INOUE, M. T.; GALVAO, F.; TORRES, D. V. Estudo ecoûsiologico sobre Araucaria angustifolia (Bert.) O. Ktze.: Fotossintese em dependencia a luz no estagio juvenil. Revista Floresta, v.10, p.5-9, 1978.

IOB, G.; VIEIRA, E. M. Seed predation of Araucaria angustifolia (Araucariaceae) in the Brazilian araucaria forest: inûuence of deposition site and comparative role of small and 'large' mamals. Plant Ecology, v.198, p.185-196, 2008.
KLEIN, R. M. Mapa Fitogeográûco do estado de Santa Catarina. Flora ilustrada catarinense. Itajaí: Herbário Barbosa Rodrigues, 1978. p.1-24.

KLEIN, R. M. Ecologia da ûora e vegetação do vale do Itajaí (continuação). Sellowia, v.32, p.165-389, 1980.

KLEIN, R. M. Aspectos dinamicos da vegetacao do sul do brasil. Sellowia, v.36, n.1, p.5-54, 1984.

KURASZ, G. et al. Caracterização do entorno da Reserva Florestal Embrapa/Epagri de Caçador (SC) usando imagem Ikonos. Floresta, v.38, p.641-649, 2008.

MANTOVANI, A.; MORELLATO, L. P. C.; REIS, M. S. Fenologia reprodutiva e produção de sementes em Araucaria angustifolia (Bert.) O. Ktze. Revista Brasileira de Botânica, v.27, n.4, p.787-796, 2004.

MATTOS, P. P. et al. Crescimento de Araucaria angustifolia na Reserva Florestal Embrapa Epagri, Caçador, SC. Pesquisa Florestal

Brasileira, v.55, p.107-114, 2007.

MELLO-FILHO, J. A.; STOEHR, G. W. D.; FABER, J. Determinação dos danos causados pela fauna a sementes e mudas de Araucaria angustifolia (Bert.) O. Ktze nos processos de regenarção natural e artiûcial. Revista Floresta, v.12, n.1, p.26-43, 1981.

MONTALDO, P. R. La bio-ecologia de Araucaria araucana (Mol) Koch. Boletin del Instituto Forestal Latino Americano de Ivestigacion e Capacitacion, v.46, p.3-55, 1974.

MULLER, J. A.; MACEDO, J. H. P. Notas preliminares sobre danos causados por animais silvestres em pinhões. Revista Floresta, v.11, n.2, p.35-41, 1980.

NARVAES, I. S.; BRENA, D. A.; LONGHI, S. J. Estrutura da regeneração natural em ûoresta ombróûla mista na ûoresta nacional de São Francisco de Paula, RS. Ciência Florestal, v.15, n.4, p.331-342, 2005.

Revista Árvore, Viçosa-MG, v.35, n.5, p.1107-1119, 2011 
NASCIMENTO, A. R. T.; LONGHI, S. J.; BRENA, D. A. Estrutura e padrões de distribuição espacial de espécies arbóreas em uma amostra de ûoresta ombróûla mista em Nova Prata, RS. Ciência Florestal, v.11, n.1, p.105-119, 2001.

NEGRELLE, R. A. B.; SILVA, F. C. Fitossociologia de um trecho de ûoresta com Araucaria angustifolia (Bert.) O. Ktze no município de Caçador-SC. Boletim de Pesquisa Florestal, v.24-25, p.37-54, 1992.

NEGRELLE, R. R. B.; LEUCHTENBERGER, R. Composição e estrutura do componente arbóreo de um remanscente de Floresta Ombróûla Mista. Revista Floresta, v.31, n.1, p.42-51, 2001.

PALUDO, G. F. et al. Estrutura demográfica e padrão espacial de uma população natural de Araucaria angustifolia (Bertol.) Kuntze (Araucariaceae) em Santa Catarina. Revista Árvore, v.33, n.6, p.1109-1121, 2009.

PERRY, G. L. W.; MILLER, B. P.; ENRIGHT, N. J. A comparison of methods for the statistical analysis of spatial point patterns in plant ecology. Plant Ecology, v.187, n.1, p.59-82, 2006.

PINTO, S. A. A. Inûuência da diocia no diâmetro e na altura de Araucaria angustifolia (Bert) O. Ktze e suas implicações na formação de áreas de produção de sementes na região de quedas do Iguaçu estado do Paraná. Revista Floresta, v.20, n.1/2, p.1-1, 1990.

PUCHALSKY, A.; MANTOVANI, M.; REIS, M. S. Variacao em populacoes naturais de Araucaria angustifolia (Bert.) O. Kuntze associada a condicoes edafo climaticas. Scientia Forestalis, v.70, p.137-148, 2006.

R DEVELOPMENT CORE TEAM. R: A Language and Environment for Statistical Computing. Vienna, Austria, 2008. ISBN 3-900051-07-0. Disponível em: <http:// www.R-project.org>.

REITZ, R.; KLEIN, R. M. Araucariáceas. Flora Ilustrada Catarinense. Itajaí: Herbario Barbosa Rodigues, 1966.
REITZ, R.; KLEIN, R. M.; REIS, A. Projeto

Madeira de Santa Catarina. Itajaí: Herbário Barbosa Rodrigues, 1978. 378p.

RIBEIRO, M. C. et al. The Brazilian Atlantic Forest: How much is left, and how is the remaining Forest ditributed? Implications for conservation. Biological Conservation, v.142, p. 1141-1153, 2009.

RIGG, L. S. Disturbance processes and spatial patterns of two emergent conifers in new caledonia. Austral Ecology, v.30, p.363-373, 2005.

RIPLEY, B. D. Modelling spatial patterns. Journal of the Royal Statistical Society, v.39, n.2, p.172-212, 1977.

RIZZINI, C. T. Plantas do Brasil: Árvores e madeiras úteis do Brasil - Manual de dendrologia brasileira. São Paulo: Edgard Blucher, 1978.

ROWLINGSON, B.; DIGGLE, P. Splancs: spatial point pattern analysis code in s-plus.

Computers and Geosciences, v.19, p.627655, 1993.

SAMPAIO, M. B.; GUARINO, E. S. G. Efeitos do pastoreio de bovinos na estrutura populacional de planta em fragmento de Floresta Ombróûla Mista. Revista Árvore, v.31, n.6, p.1035-1046, 2007.

SANQUETTA, C. R. et al. Sobrevivência de mudas de Araucaria angustifolia perante o controle de taquaras (Bambusoideae) no Paraná, Brasil. Revista Floresta, v.35, p.127-135, 2005.

SCHAAF, L. B. et al. Alteração na estrutura diamétrica de uma floresta ombrófila mista no período entre 1979 e 2000. Revista Árvore, v.30, n.2, p.283-295, 2006.

SEGER, C. D. et al. Levantamento ûorístico e análise ûtossociológica de um remanscente de Floresta Ombróûla Mista localizado no município de Pinhais, Paraná-Brasil. Revista Floresta, v.35, n.2, p.291-302, 2005.

SILVA, J. A. et al. Phytosociolgical survey in brazilian Forest Genetic Reserve of Caçador. Plant Ecology, v.133, n.1, p.1-11, 1997. 
SILVA, C. V.; REIS, M. S. Produção de pinhão na região de Caçador, SC: Aspectos da obtenção e sua importância para comunidades locais.

Ciência Florestal, v.19, n.4, p.363-374, 2009.

SOARES, R. V. Consideracoes sobre a regeneracao natural da Araucaria angustifolia. Revista Floresta, v.10, n.2, p.12-18, 1979.

SOUZA, A. F. Ecological interpretation of multiple population size structures in trees: The case of Araucaria angustifolia in south america. Austral Ecology, v.32, p.524-533, 2007.
SOUZA, A. F. et al. Regeneration patterns of a long-lived dominant conifer and the effects of logging in southern South America. Acta Oecologica, v.34, p.221-232, 2008.

VEBLEN, T. T. Regeneration patterns in Araucaria araucana forests in chile. Journal of Biogeography, v.9, n.1, p.11-28, 1982.

ZAR, J. H. Biostatistical analysis. 4.ed. New Jersey: Prentice-Hall Internacional, 1999. 
